\title{
AUDIENCE RESPONSE SYSTEMS: BENEFITS \& UTILIZATION
}

\author{
Emília PIETRIKOVÁ \\ Department of Computers and Informatics, Faculty of Electrical Engineering and Informatics, Technical University of Košice, \\ Letná 9, 04200 Košice, tel. 055/602 2554, E-mail: emilia.pietrikova@tuke.sk
}

\begin{abstract}
The following article deals with Audience Response Systems (ARS). The main goal is to provide a survey on utilization possibilities of ARS in various forms, with a focus on particular advantages. The article is dedicated to those readers who interact with an audience or a larger group of people. Particular attention is paid to technologies available to construct one's own ARS, whether as a combination of existing and custom solutions, or only one's own solution. Separate part deals with an experimental comparison of two of the available technologies. Combination of Arduino and Java as a combination of hardware and software on one hand, and combination of PHP and web on the other hand. The conclusion includes an overall evaluation of the tested solutions.
\end{abstract}

Keywords: Audience Response System, survey, Arduino, Java, PHP, web, Microsoft Mouse Mischief

\section{INTRODUCTION}

Modern educational techniques, focusing primarily on academic performance, require more effort from lecturers as well as from students. Classic lectures and individual consultations which used to be the only source of knowledge, have been suppressed and nowadays, even modern technologies such as Internet or multimedia are no longer the predominant teaching means. At many universities, lectures have become a place where lecturers and students are wishing to learn more effectively [1].

Audience Response Systems (ARS) offer a new concept for development of interaction between a lecturer and students. Tools that are part of these systems bring many interesting interactions and provide a rapid feedback to the teacher about how students understand the given problem [2]. Since ARS provides information towards students as well, such a feedback can be also very important part of student assessment. This can be the information regarding their knowledge and possible suggestions for an improvement. This technology, respectively method of interaction between students and lecturers, allows [3]:

- lecturers to ask their students questions,

- students to enter their answers using separate devices,

- both lecturers and students to get feedback.

The following sections provide an analysis of selected ARS devices and an experimental comparison of available technologies through an implementation of a web-based ARS and a digital ARS device based on Arduino platform.

The goal is to provide a survey on existing ARS solutions and technologies available to construct one's own ARS. Primarily, ARS consists of transmitter, receiver and data processing applications. Two technologies are compared as a verification of two of the available technologies. Hardware (digital device) solution is based on Arduino technology in cooperation with Java application and it may represent a simple ARS solution. An alternative to classic ARS can be a web-based tool used for an online research.

\section{AUDIENCE RESPONSE SYSTEMS}

ARS used in the academic field have developed an interactive way of communication between lecturers and students. Many lecturers claim it has helped to increase student engagement, thereby obtain a quick feedback [2] [3]. Such a method enriches the entire academic course. There are technological and non-technological ARS solutions. The classic show of hands is often replaced by modern instruments. In recent years, engineers have developed various electronic systems enabling the use of voting devices as a transmitter and receiver (see Fig. 1). ARS comprises hardware voting device, however, it also includes software for data processing and evaluation of after the voting process.

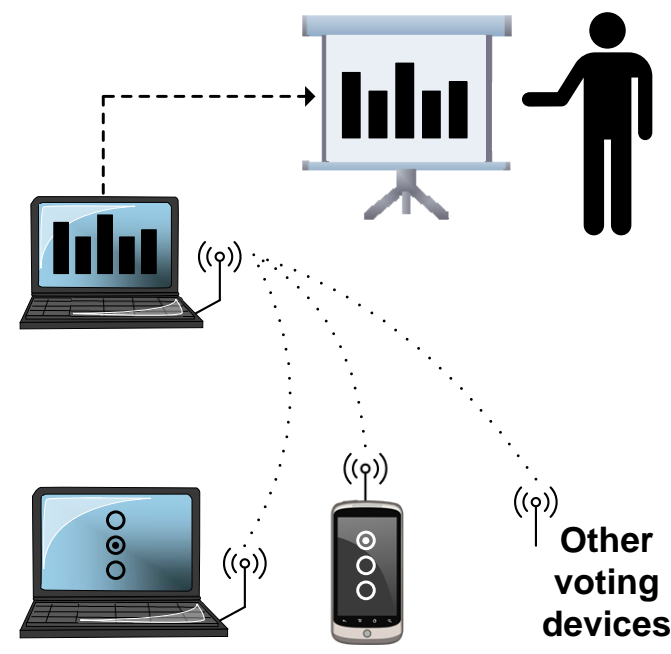

Fig. 1 Architecture of standard Audience Response System (ARS)

Though ARS is the most popular, researchers have come up with several alternative names: Personal Response System (PRS), Electronic Voting System (EVS), Student Response System (SRS), Classroom Performance Systems (CPS) [1]. 


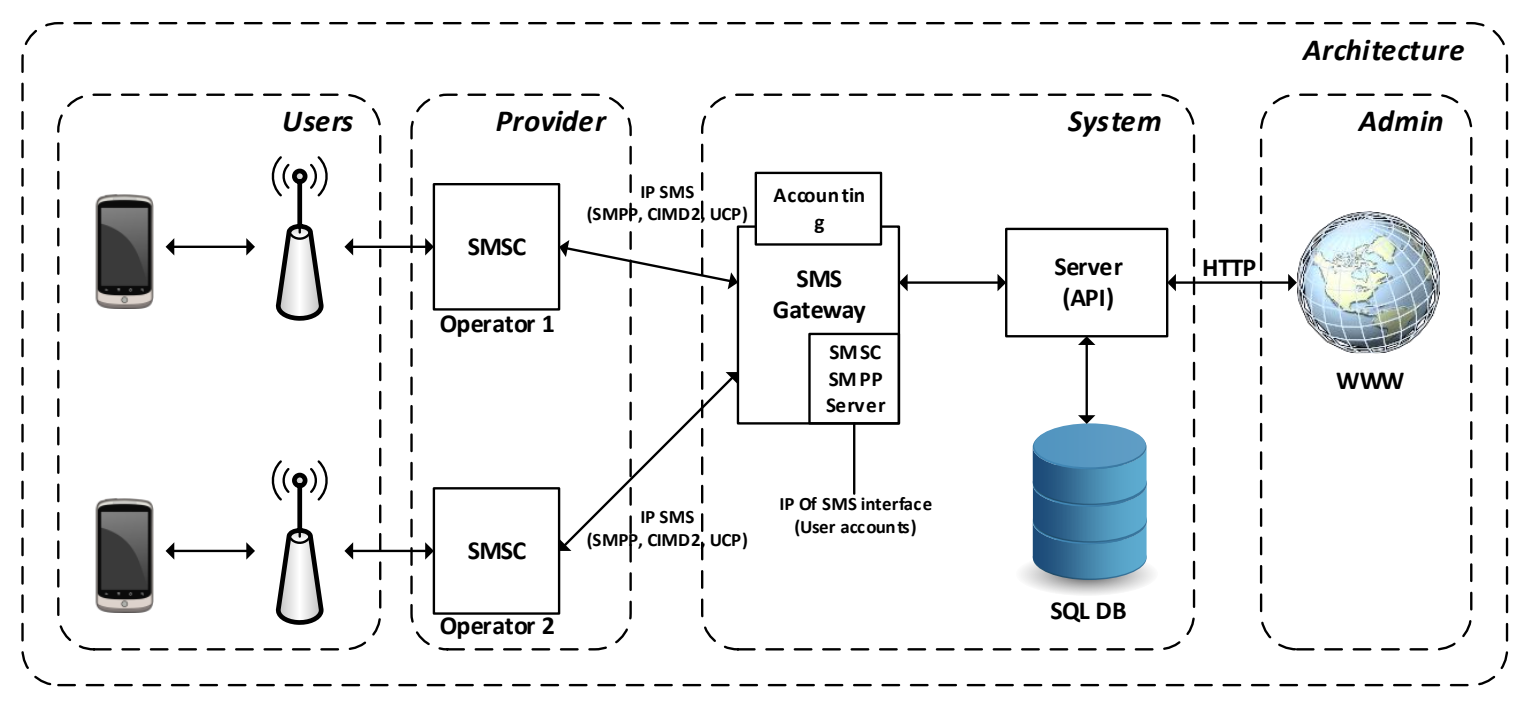

Fig. 2 Architecture of ARS using sms gateway

There are several ways of how to introduce ARS. One is the purchase of existing (commercial) electronic devices providing possibilities to vote and to obtain an immediate feedback, usually as a graph with voting results. In most cases, such systems are expensive, since they require to purchase multiple voting devices along with a receiver and a software (for graphical interpretation of voting results). One the other hand, several solutions are offered for free as an extension to other software, mostly as plugins for Microsoft Office. Then, the client pays only for the hardware.

An alternative solution uses mobile devices and sms (see Fig. 2). However, this solution has been deprecated due to rapid development of other technologies.

Another possibility is to construct one's own system. As for the cost, it represents a much cheaper way of introducing ARS. There are various technologies for transfering data between devices, their processing and evaluation. Conventional infrared systems, radio systems or Wi-Fi wireless communications belong to standard data transmission capabilities between voters and a receiver.

An appropriate choice of hardware technology is an important step when constructing one's own ARS. Since the majority of voting solutions are electronic devices, suitable selection should move to this direction (IR or RF). There are a lot of electronic components through which it would be possible to construct a high quality transmitter and receiver. If the selection is facing slightly different direction (data transfer via Wi-Fi technology) one should pay attention to the fact that the voting system would be equipped by mobile phones, laptops or tablets. In such a case, it is not necessary to construct a physical transmitter. One should consider that for such devices only voting software is necessary, processing and evaluating the data.

One possible alternative can be a web solution. Such a solution does not require physical transmitter nor receiver. However, besides computers, tablets or mobile phones, this solution requires a web server.

As for the RF transmission, transmitter and receiver are electronic components where ensuring mutual communication represent an important role. With Wi-Fi wireless transmission, it is necessary to ensure communication over the Internet using correct web services and communication protocols. When implementing a software for data processing, the choice of technology depends on a hardware on which it will run and also it depends on a technology for data transmission. In the RF transmission, there are unlimited possibilities. Application for data processing can be created through various programming languages. It depends on experience of a programmer. With $\mathrm{Wi}-\mathrm{Fi}$, the realization is slightly limited. Since mobiles devices run on different operating systems, one should consider appropriate platforms and corresponding programming languages. Each of the mentioned possibilities has both advantages and disadvantages that need to be taken into consideration.

Today, a lot of manufacturers use different technologies and methods to achieve the most effective solution. Turning Technologies is represented by a full range of products. Since they meet most needs of educational environments, these products are the most popular among many lecturers. The less well-known (but financially more advantageous) are eInstruction, $i>$ clicker, SMART Technologies, or Quizdom. Keypoint Interactive includes complete solution in the field of ARS directly integrated into the Microsoft PowerPoint. Market survey performed within this study paid a separated attention to price range of these solutions. The result is showed in Tab. 1

Number of well-known universities already implemented ARS in their educational systems, e.g. East Carolina University, Liberty University, The University of Utah, University of Winconsin-Madison, University of Illinois, University of East Anglia, RMIT University, University of Kansas Medical Center, Harvard Kennedy School, Boston University, and many others.

Most of these universities included ARS in their Regulations [4] [5] and their students are required to purchase their own voting tools [6] [7]. 
Table 1 Survey on prices of most popular brands

\begin{tabular}{|l|c|c|c|}
\hline Brand & Receiver & Transmitter & Set \\
\hline \hline $\begin{array}{c}\text { Keypoint } \\
\text { Interactive }\end{array}$ & $75 €$ & $33 €$ & $468 €$ \\
\hline $\begin{array}{c}\text { Turning } \\
\text { Technologies }\end{array}$ & $124 €$ & $42 €$ & - \\
\hline eInstruction & $71 €$ & $32 €$ & - \\
\hline $\begin{array}{c}\text { i>clicker } \\
\text { SMART } \\
\text { Technologies }\end{array}$ & - & $45 €$ & - \\
\hline Quizdom & $94 €$ & $52 €$ & - \\
\hline
\end{tabular}

\section{EXPERIMENT}

As already mentioned, most ARS tools consist of hardware (voting devices) and software (data collection and evaluation). Each hardware requires a financial investment and each solution has a different complexity. This is why one may consider more variations and select the most appropriate one. Within our experiment we analyzed two of the cheaper existing solutions. Moreover, we have chosen to perform our own implementation of ARS as well, using two of the available technologies.

\subsection{Keypoint Interactive}

First part of our experiment was focused on utilization of Reply system by Keypoint Interactive, tested several times during freshman university courses in the area of Informatics. Regarding the usage, direct experience showed both software and hardware are intuitive. Regarding the effect, 80 students expressed their positive attitude, summarized in Tab. 2. Besides anonymous opinion collector, the system was used as attandance tracker or immediate assessment tool as well.

Table 2 Feedback on student opinion after first and second experience with ARS

\begin{tabular}{|l|c|c|}
\hline Question & Yes & No \\
\hline \hline Do you like ARS? & $87 \%$ & $13 \%$ \\
\hline $\begin{array}{r}\text { Would you like to work } \\
\text { with ARS in the future? }\end{array}$ & $96 \%$ & $4 \%$ \\
\hline
\end{tabular}

There are four main drawbacks of purchased systems: Price (see Tab. 1), only 1 correct answer, software dependent from one platform (MS Windows with MS Office), software may not meet lecturer's requirements.

In our experiment, the problem with one platform dependency was solved by using virtual machine $[\overline{8}]$. The problem with only one correct answer has been identified by several authors. They claim lecturers should have more possibilities to ask questions and collect answers in order to identify what students actually know and not what they guessed or deduced. To solve this problem, we would need to purchase more expensive system with multiple-choice transmitters.

\subsection{MS Mouse Mischief}

MS Mouse Mischief (MMM) is an extension to MS PowerPoint (version 2007 and higher) downloadable for free. As for the system requirements, most classrooms are equipped with modern hardware devices with an appropriate software [9]. These include desktop computers, usb hubs and a multimedia projector where MS PowerPoint is the required software.

After successful installation, one may prepare questions and multiple answer choices. As a voting device, computer mouse (available both wired and wireless technologies) is used while questions and answers are displayed through the projector connected to a central computer. Wired technology requires usb hub devices connecting all the mice and a central computer, wireless technology requires a receiver (see Fig. 3).

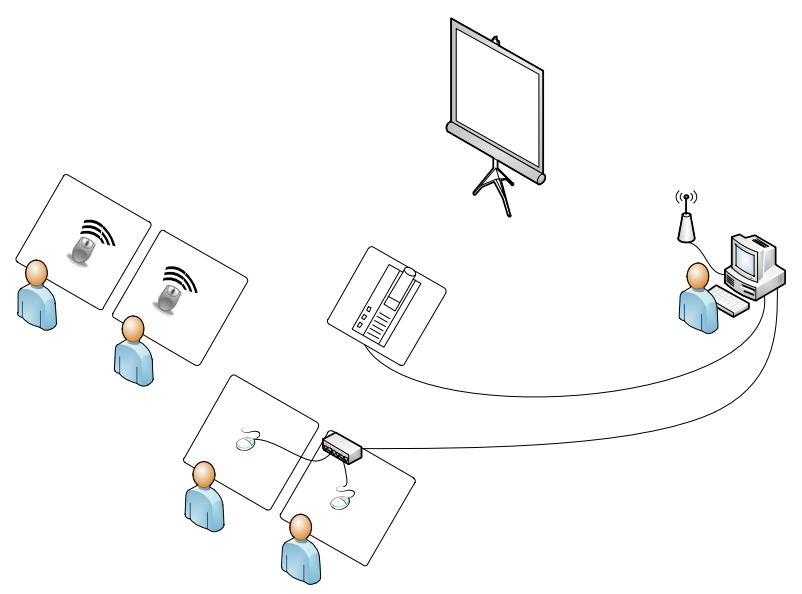

Fig. 3 Architecture of MS Mouse Mischief deployment

Assuming the classroom has an elementary equipment, one may invest from 70 to $130 €$ (depends on manufacturer and the product quality).

\subsection{ARS and Arduino Platform}

Arduino is an open source platform with the core of ATmega microcontroller by Atmel. To write a code, one may use graphical development environment Arduino IDE (free). Arduino allows construction of a variety of interactive tools, obtaining data from sensors and switches, controlling the motors, lights etc. Each project works independently or in combination with computer software. Elementary Arduino board can be constructed manually or it can be already prebuilt. There are several similar Arduino plat- 
forms providing comparable functionality. The main reasons for selecting this platform were [10]:

- low cost,

- multiplatform (runs on Windows, Linux and Mac OS),

- simple, clear programming environment suitable for beginners and flexible for experienced users,

- open source and extensible software - experienced programmers have the option of extending the tool by appropriate $\mathrm{C}$ libraries.

Currently, the market offers number of Arduino products (motherboards, add-on modules and various sets). Transmitter and receiver are usually two different devices using wireless technology for data transmission. It is necessary to construct the main receiver and several transmitters (voting devices). Base on the current knowledge and appreciation of transfer data technology, the most appropriate technology for wireless communication between Arduino devices is technology using radio transmission.

When selecting suitable components, one should consider energy consumed by particular components: a number of transmitters and one receiver. Other important factors are signal range, transmission speed, support of addressing while sending a message, price, functionality, and settings complexity [12]. The following list contains components selected for the experiment:

- Iteaduino UNO development board,

- Arduino Wireless SD Shield (XBee),

- XBee Series 2,

- connector,

- breadboard,

- buttons,

- resistors,

- batteries with charger for batteries,

- USB cable A-B micro.

The complete ARS consist of hardware and software. Hardware part consist of transmitter and receiver, constructed from the mentioned digital components, enriched by the software part. Software part consists of a program which activates the hardware and an application for processing and evaluation of the collected data. The application should run independently on the computer to which the receiver is connected. The receiver collects responses from different transmitters and sends them to the serial port of the computer. The aim of this application is to read data from the corresponding PC serial port and to evaluate them. Then, the user will see graph with corresponding results.

As a result, we experimentally constructed one receiver and two transmitters. Although the price was lower than the price of purchased systems, the implementation might be difficult. However, platforms like Arduino make the construction less difficult. Moreover, one has a chance to create a custom extension to MS Office or a standalone application meeting requirements of a particular lecturer.

\subsection{ARS and Web}

Web-based system represents a tool for online voting. This solution is an excellent alternative to classic voting via digital devices. For experimental purposes, we decided to use PHP language in combination with HTML and CSS, relying on statistics of the most used programming languages for creating web-based applications.

This solution has proved to be the least expensive (almost for free). It is simple to use: The users should only have their own device with a web browser and a link to the poll (e.g. in form of a qr-code). The only problem is the user interface design. Web should be attractive and intuitive to use. This does not seem to be too difficult, however, we have experienced several uncertainties regarding the design of an attractive but simple-to-use user interface. This is why we recommend to use some of existing web portals, such as sli.do or surveymonkey.com, if one has decided to use web solution.

\section{CONCLUSION}

Generally, ARS are tools raising interactivity between lecturers and audience. ARS can be helpful when getting feedback on understanding or interest [13], or when collecting and representing questionnaire data [11]. Positive side-effects usually include tracking of attendance or holding listeners' attention. Many authors have already claimed that ARS has a significant impact on knowledge tranfer in higher education, especially in medical area [2] [4]. However, most of them do not deal with financial issues neither with multiplatform deployment. Our study utilized ARS within IT lectures in various forms and proved the system to be helpful in this area as well.

Lecturers may purchase ARS, choosing from large variety of commercial solutions. However, these solutions are quite expensive, particularly if coming from financially insufficiently equipped environment. Our investigation has showed current price range varies from 500 to $1000 €$ for a smaller class (up to 30 students).

In such cases, lecturers should draw their attention to less expensive solutions. One of such solutions is MS Mouse Mischief, providing access to this technology even if limited in the resources. Students do not need their your own equipment but computers and mice installed in the room. Unlike other systems, computer mice are relatively cheap and available equipment. This is why we consider this solution as a cost effective alternative to other expensive systems. With several USB hubs and computer mice (wired or wireless), students can interact with lecturer's presentation.

Another way can be construction of one's own ARS using wireless communication. The analysis shows that the most appropriate option is radio communication. Each technology has both advantages and disadvantages regard- 
ing the difficulty of implementation. Although radio communication uses slow data transfer, its power consumption is less as opposed to the Wi-Fi technology. Financially, Wi-fi modules are more expensive than radio-transmission equipment. In our experiment, Arduino has proved to be an open source platform providing simple and clean programming environment, being multiplatform and cheap. It has also proved to be platform designed for quick and easy construction. As a result, variety of interactive devices have been constructed.

Our experiment included web technologies as well, using a web site as a tool for online survey. This solution was the least expensive, however, the most challenging regarding the design of user interface. Since there are already a lot of similar solutions available on the Internet and most of them are free, we recommend to use existing web solutions instead of preparing custom web solution.

As a result, we highly recommend to use any variant of ARS when teaching and learning. Such an approach attracts student attention and makes knowledge transfer more effective. Moreover, if it is appropriately combined with other innovative methods [14], the overall result might be surprising.

\section{ACKNOWLEDGEMENT}

This work was supported by project KEGA No. 019TUKE-4/2014 Integration of the Basic Theories of Software Engineering into Courses for Informatics Master Study Programmes at Technical Universities - Proposal and Implementation.

\section{REFERENCES}

[1] SMITH, M.K. - WOOD, W.B. - ADAMS, W.K. WIEMAN, C. - KNIGHT, J.K. - GUILD, N. - SU, T.T.: Why peer discussion improves student performance on in-class concept questions, Science 323, No. 5910 (2009) 122-124

[2] LATESSA, R. - MOUW, D.: Use of an Audience Response System to Augment Interactive Learning, Family Medicine Journal 37, No. 1 (2005) 12-14

[3] WENZ, H.J. - ZUPANIC, M. - KLOSA, K. SCHNEIDER, B. - KARSTEN, G.: Using an Audience Response System to Improve Learning Success in Practical Skills Training Courses in Dental Studies - A Randomised, Controlled Cross-Over Study, European Journal of Dental Education 18, No. 3 (2014) $147-153$

[4] University of Wisconsin-Madison: UW School of Medicine and Public Health Audience Response System Policy. Available at (January 2015): http://www.med.wisc.edu/facilities/hslc/uwschool-of-medicine-and-public-healthaudience-response-system-policy/28880

[5] The University of Utah: Audience Response Systems (Clicker). Available at (January 2015): http: //ims.utah.edu/clicker/
[6] Liberty University: About Clickers \& Receivers. Available at (January 2015): http: //www.liberty.edu/informationtechnology/itsupport/index.cfm?PID $=22126$

[7] University of East Anglia: Audience Response Systems: Real Time Interaction and Participation. Available at (January 2015): https://portal.uea.ac.uk/ learning-technology/ars

[8] VOKOROKOS, L. - MADOŠ, B - ÁDÁM, N. BALÁŽ, A.: Innovative operating memory architecture for computers using the data driven computation model, Acta Polytechnica Hungarica 10, No. 5 (2013) 63-79

[9] Microsoft: Microsoft Mouse Mischief. Available at (January 2015): http://www.microsoft.com/ multipoint/mouse-mischief/en-us/default.aspx

[10] Arduino: Why Arduino? Available at (January 2015): http://arduino.cc/en/Guide/Introduction

[11] JACKOWSKA-STRUMILLO, L. - NOWAKOWSKI, J. - STRUMILLO, P. - TOMCZAK, P.: Interactive Question Based Learning Methodology and Clickers: Fundamentals of Computer Science Course Case Study, IEEE International Conference on Human System Interactions, 2013, pp. 439-442

[12] ENNERT, M. - CHOVANCOVÁ, E. DUDLÁKOVÁ, Z.: Testing of IDS model using several intrusion detection tools, Journal of Applied Mathematics and Computational Mechanics 14, No. 1 (2015) 55-62

[13] TANGEN, J. - CONSTABLE, M. - DURRANT, E. - TEETER, C. - BESTON, B. - KIM, J.: The role of interest and images in slideware presentations, Computers \& Education 56, No. 3 (2011) 865-872

[14] BIŇAS, M. - PIETRIKOVÁ, E.: Useful recommendations for successful implementation of programming courses, IEEE International Conference on Emerging eLearning Technologies and Applications (ICETA), 2014, pp. 397-401

Received October 27, 2015, accepted January 26, 2016

\section{BIOGRAPHY}

Emília Pietriková is Assistant Professor at the Department of Computers and Informatics, Technical University of Košice, Slovakia. She received her MSc in 2010 and her PhD in 2014 in Informatics from Technical University of Košice. In 2010 she spent 1 month at the Department of Telematics at Norwegian University of Science and Technology, Norway. In 2011 she spent 1 semester at the Department of Computer Architecture at University of Málaga, Spain. The subject of her research is abstraction and generation in programming languages, and quality of education. 